\section{Case Reports in Ophthalmology}

Case Rep Ophthalmol 2020;11:391-394

DOI: 10.1159/000507846

Published online: July 28, 2020
(C) 2020 The Author(s)

Published by S. Karger AG, Basel www.karger.com/cop

\title{
A Case of Pediatric Cyanoacrylate Adhesive Injury to the Eye
}

\author{
Aika Tsutsui Kazunobu Sugihara Masaki Tanito \\ Department of Ophthalmology, Shimane University Faculty of Medicine, Shimane, Japan
}

\section{Keywords}

Eye trauma $\cdot$ Superglue $\cdot$ Cyanoacrylate adhesive $\cdot$ Hook technique $\cdot$ Tarsorrhaphy

\begin{abstract}
A 5-year-old Japanese boy presented to the emergency room of our hospital because his right eye had been glued shut for $2 \mathrm{~h}$ following instillation of cyanoacrylate adhesive. The clinical examination showed redness of the right upper and lower lid skin; the right eyelid margins adhered to each other because of matting of the eyelashes, and crystallized glue was found within the matted eyelashes. The "hook" technique, which separates the eyelids using a blunttip device, was performed to separate the lids. The technique seems to be simple and safe for physicians such as ophthalmology residents to use to treat cyanoacrylate-induced adhesion of the eyelids of patients who often first present to their local emergency room as in our case.
\end{abstract}

(c) 2020 The Author(s)

Published by S. Karger AG, Basel

\section{Introduction}

Cyanoacrylate glue, a common household adhesive, is a monomer of cyanoacetate, which polymerizes within seconds via a reaction to water in the air or target surface, such as the eyelid margins and eyelashes, and results in a chemical tarsorrhaphy [1,2]. We report a case of superglue injury to the eye of a Japanese boy. 


\section{Case Reports in Ophthalmology}

\section{Case Report}

A 5-year-old Japanese child presented to the emergency room of our hospital because his right eye had been glued shut for $2 \mathrm{~h}$ following accidental instillation (mistaken as eye drops) of cyanoacrylate adhesive (Aron Alpha ${ }^{\circledR}$, Toagosei Company, Ltd., Tokyo, Japan) by himself. Before presentation, the parents attempted to open the eyelids with their fingers and a lid wash of warm water at home, and an emergency room doctor at his local hospital failed to separate the eyelids. The clinical examination showed redness of the right upper and lower lid skin; the right eyelid margins adhered to each other because of matting of the eyelashes, and crystallized glue was found within the matted eyelashes (Fig. 1a).

The "hook" technique was performed to separate the lids [2] (Fig. 1b). After a topical anesthetic $(0.4 \%$ oxybuprocaine, Benoxil ophthalmic solution, Santen Pharmaceutical, Osaka, Japan) was instilled through a gap in the lid fissure, an ophthalmic ointment $(0.3 \%$ ofloxacin, Taribid ophthalmic ointment, Santen Pharmaceutical) was applied to moisten the crystallized glue particles. With parental permission, the patient was restrained by wrapping him in a blanket. A blunt-tip forceps (S-325, Beaupre epilation forceps, Inami \& Co. Ltd., Tokyo, Japan) was inserted through the gap, and the tip was moved parallel to the lid margins to the adhesion site. The tip was moved slightly upward and away from the ocular surface to minimize contact between the instrument and the ocular surface. Counterpressure opposite to the forceps movement was applied with the fellow hand. The lid margins were separated successfully; except for mild conjunctival congestion, slit-lamp examination showed no ocular surface injury (Fig. 1c). The patient was prescribed 1.5\% levofloxacin eye drops (Nipro, Osaka, Japan) 4 times/day. Two months later, the residual glue had sloughed off (Fig. 1d); no additional complications developed.

\section{Discussion/Conclusion}

After the initial case of cyanoacrylate eye injury reported in the early 1980s [3], other cases have been reported [4-8]. However, the report of a superglue episode involving an eye in Japan is unique in the literature. Outside of Japan, the superglue packaging resembles ophthalmic medication eyedropper bottles and has been associated frequently with accidental application of cyanoacrylate to eyes and eyelids [1]. The packaging used by Aron Alpha ${ }^{\circledR}$, a major brand of cyanoacrylate adhesive in Japan, differs markedly from eyedropper bottles (Fig. 1e), which explains the few reports of superglue ocular injury in Japan, suggesting the critical role of the package design in the development of ocular superglue injury.

Strategies to manage superglue episodes have included observation [4], manual forceful lid opening, overnight wet patching [6], chemical dissolution with acetone [7], and lash trimming under topical [5] or general [7] anesthesia for children. The hook technique, which separates the eyelids using a strabismus surgery muscle hook [2] or any other blunt-tip device as described here, seems to be simple and safe for physicians such as ophthalmology residents to use to treat cyanoacrylate-induced adhesion of the eyelids of patients who often first present to their local emergency room as in our case. 


\section{Case Reports in Ophthalmology}

\section{Statement of Ethics}

This study adhered to the tenets of the Declaration of Helsinki. Written informed consent was obtained from the patient's guardian for publication of this case report and any accompanying pictures.

\section{Conflict of Interest Statement}

The authors have no conflicts of interest for this study.

\section{Funding Sources}

No financial support was provided.

\section{Author Contributions}

A.T., K.S., and M.T. treated the subject and collected the clinical data. M.T. wrote the manuscript, and A.T. and K.S. revised the manuscript. All authors approved the final version of the manuscript. The authors agree to be responsible for all aspects of this work.

\section{References}

1 Desai SP, Teggihalli BC, Bhola R. Superglue mistaken for eye drops. Arch Dis Child. 2005 Nov;90(11):1193.

2 Jijelava K, Le H, Parker J, Yee J. Getting hooked: a simple technique for the treatment of adhesive injuries to the eyelids. J Emerg Med. 2017 Jan;52(1):74-6.

3 Margo CE, Trobe JD. Tarsorrhaphy from accidental instillation of cyanoacrylate adhesive in the eye. JAMA. 1982 Feb;247(5):660-1.

4 McLean CJ. Ocular superglue injury. J Accid Emerg Med. 1997 Jan;14(1):40-1.

5 Pujari A, Shakrawal J, Gagrani M, Bajaj MS. Paediatric ocular super glue injuries: assessment of two cases. BMJ Case Rep. 2018 Sep;2018:bcr-2018-226198.

6 Raynor LA. Treatment for inadvertent cyanoacrylate tarsorrhaphy: case report. Arch Ophthalmol. 1988 Aug;106(8):1033.

7 Reddy SC. Superglue injuries of the eye. Int J Ophthalmol. 2012;5(5):634-7.

8 Tabatabaei SA, Modanloo S, Ghiyasvand AM, Pouryani A, Soleimani M, Tabatabaei SM, et al. Epidemiological aspects of ocular superglue injuries. Int J Ophthalmol. 2016 Feb;9(2):278-81. 

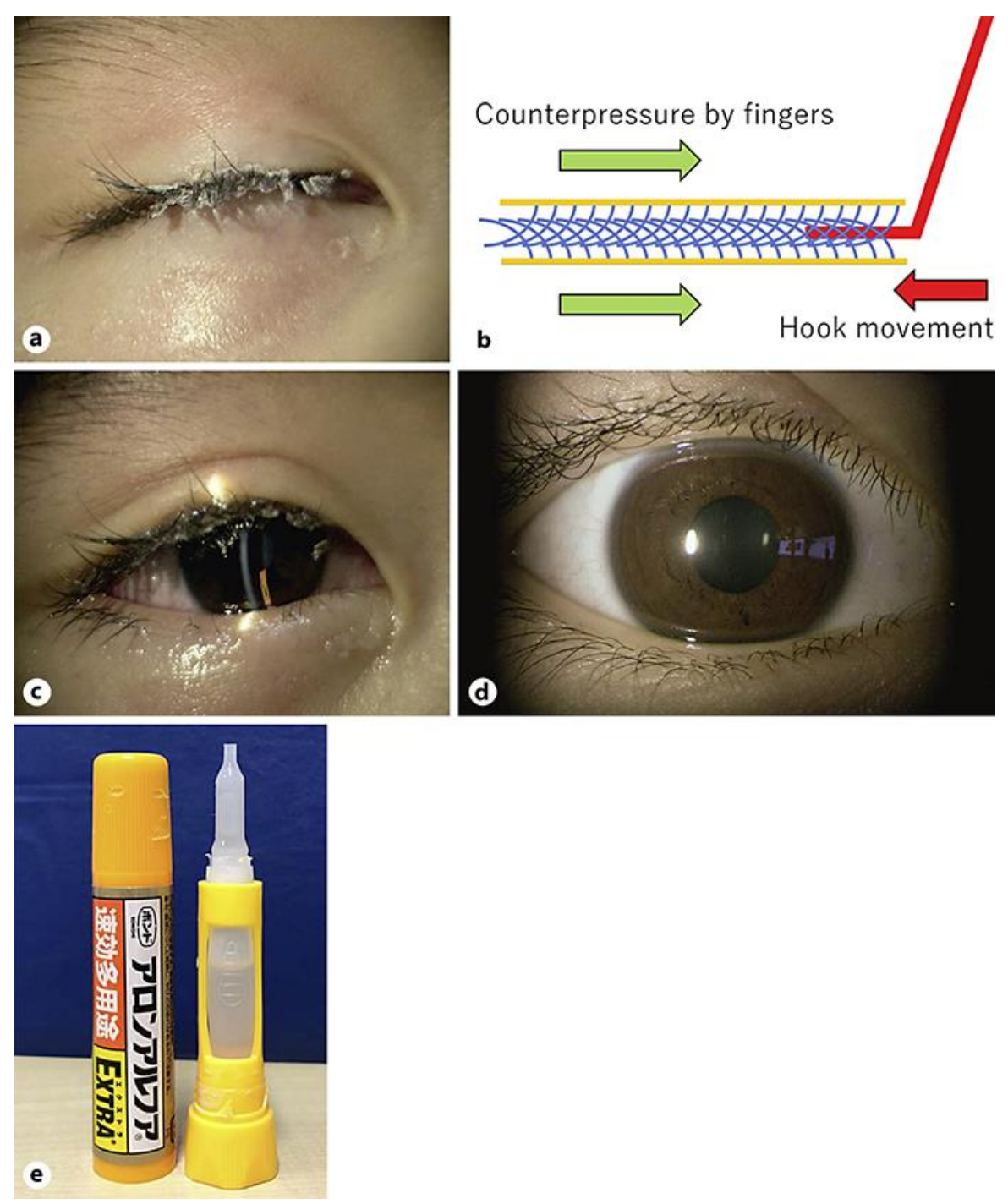

Fig. 1. Peri-treatment findings. a At referral, the upper and lower lid margins adhered to each other after inadvertent instillation of cyanoacrylate adhesive in the patient's right eye. $\mathbf{b}$ Schematic drawing of the "hook" technique. Yellow lines and blue curves indicate lid margins and matted eyelashes, respectively. c The eyelids were separated using the "hook" technique with a dull-tip forceps. d By 2 months, the remaining glue had disappeared. e The bottle of the cyanoacrylate adhesive that was involved in this case. 